\title{
Patterned Electroplating of Micrometer Scale Magnetic Structures on Glass Substrates
}

\author{
Anthony H. B. de Vries, Johannes. S. Kanger, Bea E. Krenn, and Roel van Driel
}

\begin{abstract}
This paper has developed a new method of micro patterned electroplating that enables the fabrication of micrometer scale magnetic structures on glass substrates. In contrast to other methods, the process as developed here leaves the surrounding substrate area untouched: that is there is no seed layer present except underneath the magnetic structures. Patterned cobalt layers of thicknesses up to $8 \mu \mathrm{m}$ were fabricated exhibiting high saturation $(1.5 \mathrm{~T})$ and low coercive force. These layers have been used to create micrometer scale magnetic poles (magnetic tweezers) for biological and biophysical applications, where a clean untouched glass substrate in the sample area is most important. The technique, however, can be used in any situation where a residual seed layer on the surrounding area is unwanted, and afterwards removal of this seed layer is difficult or impossible.

[1094]
\end{abstract}

Index Terms-Cobalt, electroplating, glass, lift-off photolithography, magnetic tweezers, pyrex wafer.

\section{INTRODUCTION}

A $\mathrm{N}$ exiting new field of biophysical research that is starting to develop is to measure and exert directed forces on individual probes and molecules inside living cells. Present day applications concentrate on the measurement of the (local) viscoelastic properties of cells. A large variety of techniques can be used, like magnetic bead microrheometry [1], optical tweezers (OT) [2], atomic force microscopy (AFM) [3], cell poking elastometry [4], twisted magnetic bead relaxation [5] and magnetic tweezers [6], [7]. These techniques, although very successful, have limited application. In order to exert biological relevant forces, relatively large actuators (several micrometers: AFM tip, OT beads) are needed that strongly interfere with the cell and/or cell function under study. Further development in this area should therefore focus on the downsizing of the actuator. A nanometer scale actuator still able to exert biological relevant forces will open new approaches toward analysis and manipulation of molecular systems, including the genome, in live cells. An example is to assess the forces generated by motor proteins like myosin that is used for transportation of intracellular components. In addition such small probes can be functionalized to be used as biosensors to monitor local chemical and physical processes in cells and tissues and to

Manuscript received July 1, 2003; revised November 25, 2003. This research was supported by the Life Sciences Foundation (SLW), which is subsidized by the Netherlands Organization for Scientific Research (NWO). Subject Editor A. J. Ricco.

A. H. B. de Vries and J. S. Kanger are with the Institute for Biomedical Technology (BMTI), University of Twente, 7500 AE Enschede, The Netherlands (e-mail: J.S.Kanger@tnw.utwente.nl).

B. E. Krenn and R. van Driel are with the Swammerdam Institute for Life Sciences, University of Amsterdam, 1098 SM Amsterdam, The Netherlands.

Digital Object Identifier 10.1109/JMEMS.2004.828724

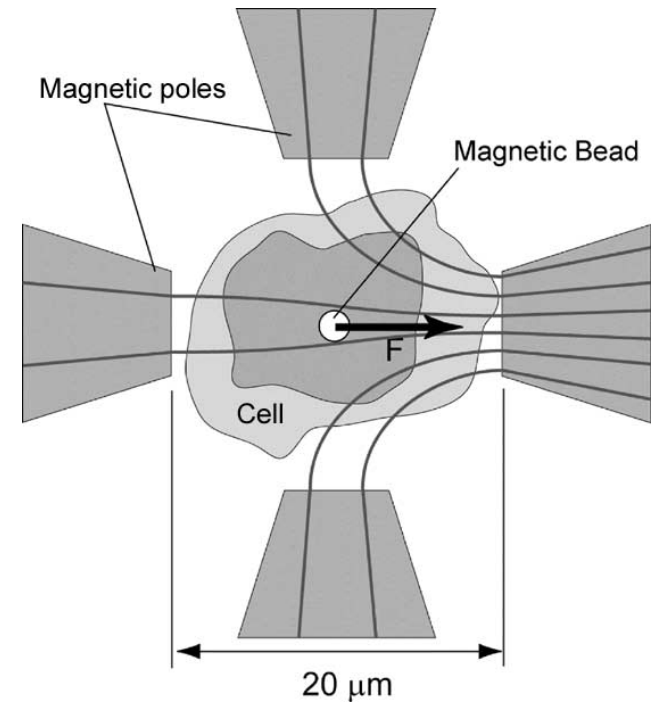

Fig. 1. The setup for a biological experiment. A living cell is positioned between magnetic poles. The pole spacing is designed to fit a single cell closely. A magnetic bead is micro-injected into the cell nucleus. A magnetic flux is generated with macroscopic coils, and transported to the pole tips, where the field lines jump over to adjacent tips to complete the loop. By having one pole transport flux into the measurement area, and the others transport it away, a geometry can be achieved with highly divergent field lines, i.e., a high field gradient. The magnetic bead will experience a force in the direction of the highest gradient. Obviously, this force can be pointing to any of the four poles, simply by changing the corresponding coil currents.

mechanically or chemically interfere with cellular processes in a highly specific manner. This will allow to obtain information on molecular processes that take place inside the cell.

Magnetic tweezers technology has the potential to exert high forces on very small beads, but it needs very small magnetic structures in order to produce the required magnetic field gradient. With such structures, directed forces could be exerted on very small beads inside living cells, as shown in Fig. 1 (see caption). The force $F$ on a magnetic bead in a magnetic field $H$ is proportional to the magnetic moment $m_{\text {bead }}$ of the bead, and the gradient of the field

$$
\vec{F}(\vec{r})=\mu_{0} \vec{m}_{\text {bead }} \cdot \nabla \vec{H}(\vec{r}) \text { with } \vec{m}_{\text {bead }}=\frac{4}{3} \pi r_{b}^{3} \vec{M}_{\text {bead }}
$$

where $\mu_{0}$ is the vacuum permeability, $M_{\text {bead }}$ the induced volume magnetization and $r_{b}$ the radius of the bead. For application to cell biology ultra small beads (radius $r_{b} \ll 1 \mu \mathrm{m}$ ) should be used in order to make the disturbance in the biological sample as small as possible and to achieve high spatial resolution. In order to achieve biological relevant forces ( $\mathrm{pN}$ range) using these small beads high field gradients are required. Realizing that the field gradient scales with the characteristic 
distance $d$ between the poles as $\nabla H \sim d^{-1}$, means that the highest forces can be obtained using micrometer scale magnetic structures. It is important to realize that also the thickness of these structures are preferably in the same range as the cell dimensions (typically $10 \mu \mathrm{m}$ ) in order to achieve homogenous and high forces.

For biological experiments, the cells and possibly the magnetic bead will typically be fluorescently labeled, and optical microscopy will be used to observe and control the experiment. Therefore, the magnetic materials that form the structures will have to be deposited on an optically transparent substrate.

Typical deposition techniques for micro patterning include evaporation and sputtering. While well controlled, these techniques are predominantly for layer thickness in the nanometer range, and ill suited for layers many micrometers high. For these thicknesses, typically electroplating would be used. While electroplating is normally done on complete unobstructed surfaces, it can also be grown in a pattern using through mask deposition. [8] With this method a removable mask is applied over a seed-layer that restricts the plating process to the desired pattern. IBM developed a variation on this called Damascene. In this case, a dielectric is first patterned, after which a seed-layer is deposited. The subsequent electro deposition is carried on until the grown layer completely overfills the whole mask. Then a planarization step is introduced, which removes all the deposited material above the mask, leaving only the small structures. The big advantage is the uniform height of all the electroplated structures, but it is obviously a very complicated process [8]-[10].

An alternative electro deposition technique is electroless plating. Here the seed layer and deposited material function as a catalyst for the electrochemical reactions. There is no power supply or electrode necessary, as the required electron supply comes from the bath itself. Electroless plating is, however, an extremely slow process, (typically a micrometer per hour) which makes it rather cumbersome for the fabrication of high structures, while also requiring very high chemical stability for the photo resist mask.

In this paper, we describe an alternative method based on electroplating to easily realize freestanding micrometer scale magnetic structures that are grown on a glass substrate and show how this method can be used to realize cobalt poles that allow exerting forces on magnetic beads. The method is such that the substrate remains optically transparent in between the magnetic structures.

\section{DeSIGN Rules}

The constraints resulting from the biological application and the requirement for high field gradient, give the design rules for the magnetic structures.

1) The working area must be optically transparent allowing (fluorescence) microcopy to visualize the experiments.

2) For proper cell attachment and biocompatibility in general, a clean transparent surface is required. Glass or plastics can both be used for growing cells.

3) To preserve the desired magnetic field geometry, the magnetic poles have to be firmly attached to the substrate. (At high fields, there will be a force attracting them toward each other)

4) The magnetic poles should be as high as possible, but not so high that there is interference with the optical pathway of a high Numerical Aperture (N.A) objective. With a pole spacing of $20 \mu \mathrm{m}$ (to fit a single cell), and a high N.A. of typically 1.2 , this corresponds to a pole height of $5 \mu \mathrm{m}$.

5) Magnetic materials should be resistant to aqueous media and should have high magnetic saturation, and preferably a low coercive force.

\section{METHOD}

\section{A. Materials}

We have chosen cobalt as the magnetic material for both its relative high magnetic saturation (1.85 $\mathrm{T}$ [11]) and its resistance to aqueous solutions. Pyrex wafers are used as a substrate.

\section{B. Micro Patterning}

The process of electroplating requires a seed layer that is connected to the cathode in the electroplating bath, and a patterned mask to define the shape of the poles that are grown.

The problem in our case is that the seed layer would cover the complete glass substrate, and is difficult to remove after electroplating. A patterned seed-layer using a lift-off process would be possible, but has the problem that the electric resistance of the lanes is not negligible. There will be substantial potential differences over the wafer, which means different grow speeds, and heights. In our case, a factor 10 to 100 difference in height between adjacent structures was observed. Also, it will be impossible to create free standing structures without seed layer current lanes connecting the structures. To solve this, we designed a "delayed lift-off" procedure.

Fig. 2 shows the complete process. Similar to normal lift-off, first a negative resist (Olin Ti-35 Image reversal resist) of $3.5 \mathrm{mi}-$ crometer thickness is used for patterning the surface (a). Then the electroplating seed layer is deposited. First a $10 \mathrm{~nm}$ titanium adhesion layer is sputtered, followed by a 200 -nm-layer of platinum (b). Platinum was used in stead of copper, for biocompatibility reasons. In contrast to normal lift-off, the resist (and metal on top of it) is not removed. This means we keep a uniform platinum layer to keep a low electric resistance across the entire wafer surface. In this way, electric potential differences between the pole tips are avoided, and we have uniform layer growth. A positive resist (Olin 908/35) of $4.5 \mu \mathrm{m}$ thickness is then applied, to pattern the platinum electroplating seed layer (c).

The electroplating bath is an aqueous solution that consists of $60 \mathrm{~g} / \mathrm{l} \mathrm{CoCl}{ }_{2} \cdot 6 \mathrm{H}_{2} \mathrm{O}, 60 \mathrm{~g} / \mathrm{H} \mathrm{H}_{3} \mathrm{BO}_{3}$ and $3.5 \mathrm{~g} / \mathrm{l} \mathrm{SDS}$. The anode is a cobalt rod of $99.9 \%$ purity. We used standard condition for the electroplating bath ( $\mathrm{pH} 3.5$, temperature $60{ }^{\circ} \mathrm{C}$ [12]). The $\mathrm{pH}$, temperature and boric acid concentration can be varied to change the phase composition (and thus magnetic properties) of the electroplated cobalt layer [13].

For electroplating it is vitally important that the surface is very clean. Just before the electroplating process, the wafer is etched for $1 \mathrm{~min}$ in oxygen plasma. This is sufficient to remove any organic compounds on the surface, but not so long 


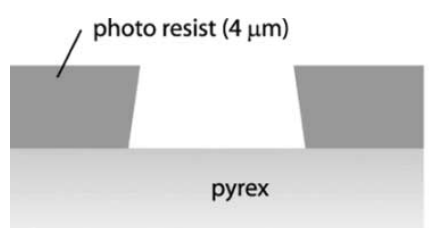

a
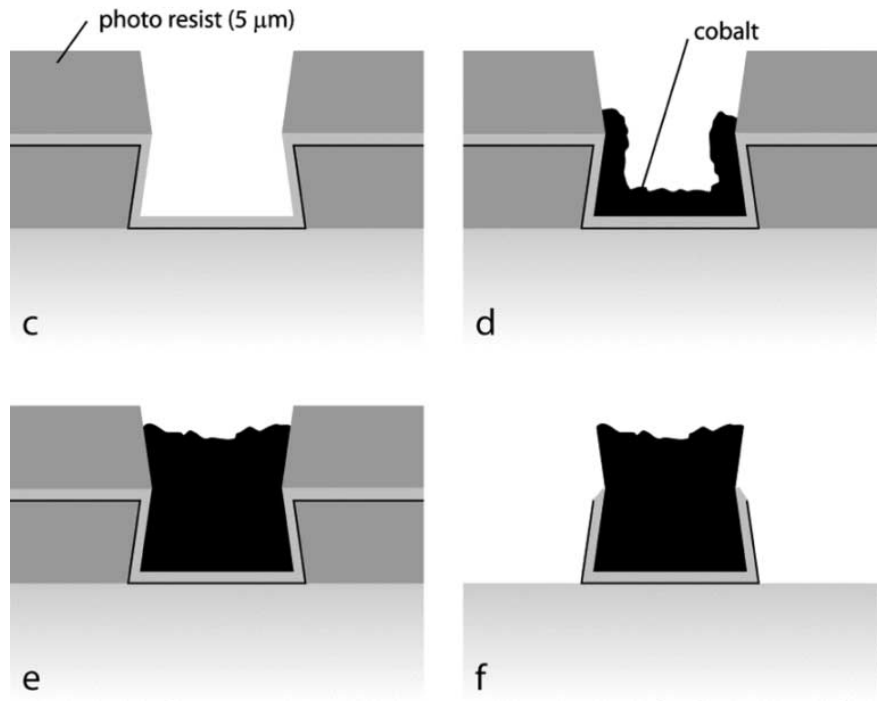

Fig. 2. Process outline. First a (negative) photo resist mask is applied on the glass substrate (a). Then the electroplating seed-layer is deposited (b). In contrast to normal lift-off, the resist layer is not removed. Instead, a second (positive) photo resist mask is applied (c). A cobalt layer is grown using electroplating (d), (e). Finally, the lift-off is performed, removing both resist layers, and the exposed seed-layer in between (f).

to significantly etch the photo resist pattern. The electroplating process is performed at a current density of $25 \mathrm{~mA} / \mathrm{cm}^{2}$. The cobalt layer will grow on all exposed platinum, so that short deposition will produce a cup-like structure with edges having the same width as the deposited layer thickness (d). When producing higher structures, this effect becomes negligible (e). If required, the second resist layer could be given a slightly smaller mask opening, to just cover the side walls of the mask, removing this effect.

After the electroplating, the lift-off is performed, by stripping both resist layers with acetone. A short treatment in an ultrasonic bath is necessary for complete removal of the resist, and tearing of the excess seed layer that was supported by it (f). We are left with free standing cobalt magnetic structures, while the glass substrate around it is devoid of any other materials.

\section{Setup for Force Measurements}

A magnetic structure of four magnetic poles has been created with the procedure described. These four poles are driven by macroscopic coils ( 80 windings) connected to a power amplifier capable of delivering a magnetomotive force of 250 ampereturns, up to a frequency of $30 \mathrm{kHz}$.

To measure the magnetic force exerted by this pole structure, we place a magnetic bead attached to a cantilever in the field, as shown in Fig. 3. We then record the bead displacement at different locations, under varied field configurations. Knowing both the magnetic moment of the bead, and the spring constant

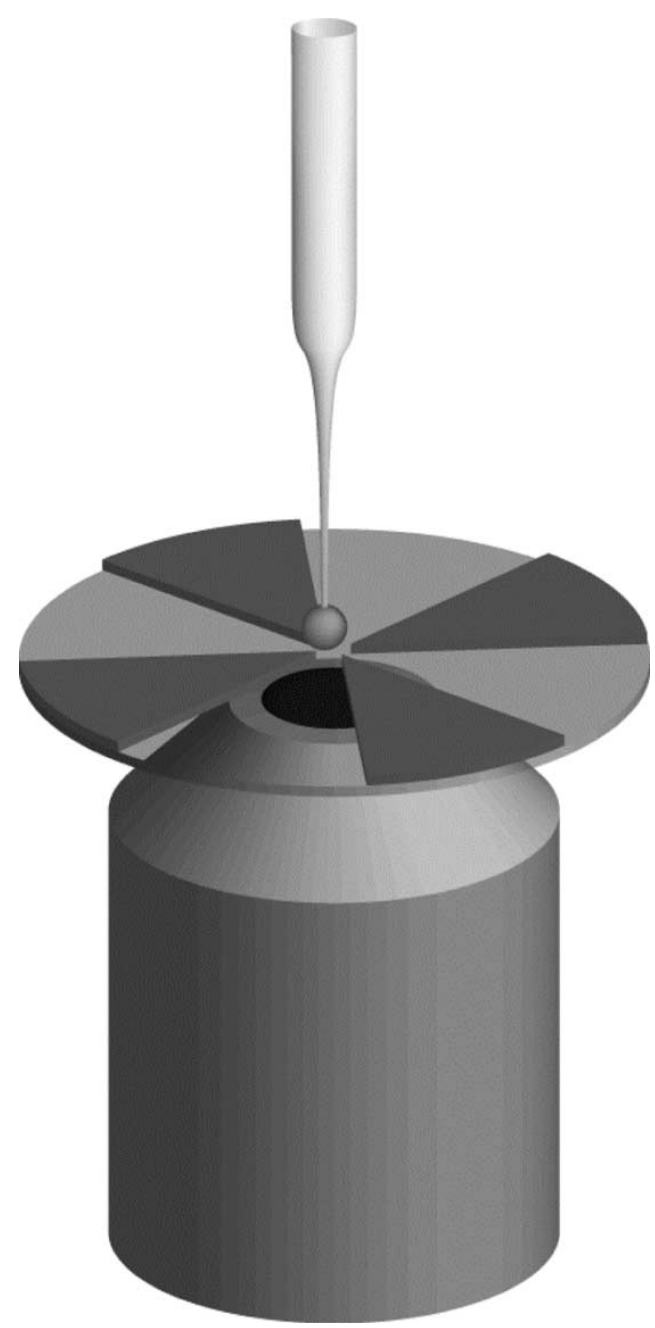

Fig. 3. The experimental setup for calibrating the magnetic forces. (Image not to scale) A magnetic bead is attached to a micropipette, and placed between the magnetic poles. The deflection due to magnetic forces is monitored with video microscopy. Knowing the magnetic moment of the bead and the stiffness of the pipette, the force on the bead, and the magnetic field gradient can be calculated.

of the cantilever, we can then directly calculate the force on the bead, and the magnetic field gradient.

Magnetic beads with a measured magnetic moment of $9 \times 10^{-13} \mathrm{~A} \cdot \mathrm{m}^{2}$ were used (Dynal Dynabead M-450, diameter $4.5 \mu \mathrm{m})$. Micropipettes were used as a cantilever. Borosilicate pipettes were pulled by a micropipette puller (P-97 Sutter). The stiffness of these pipettes was calibrated against an AFM cantilever with known spring constant. The pipettes used have spring constants between 0.3 and $2 \mathrm{nN} / \mu \mathrm{m}$. The bead deflection is observed with video microscopy and analyzed by home written image analysis software.

\section{RESULTS}

\section{A. Magnetic Structures}

Cobalt layers were fabricated using the method as described. Thickness of these layers is $3 \mu \mathrm{m}$, as measured with a Dektak surface profiler. From these measurements, a deposition rate of $0.6 \mu \mathrm{m}$ per minute was derived.

The magnetic properties of the electroplated material are characterized using a vibrating-sample magnetometer (VSM). 


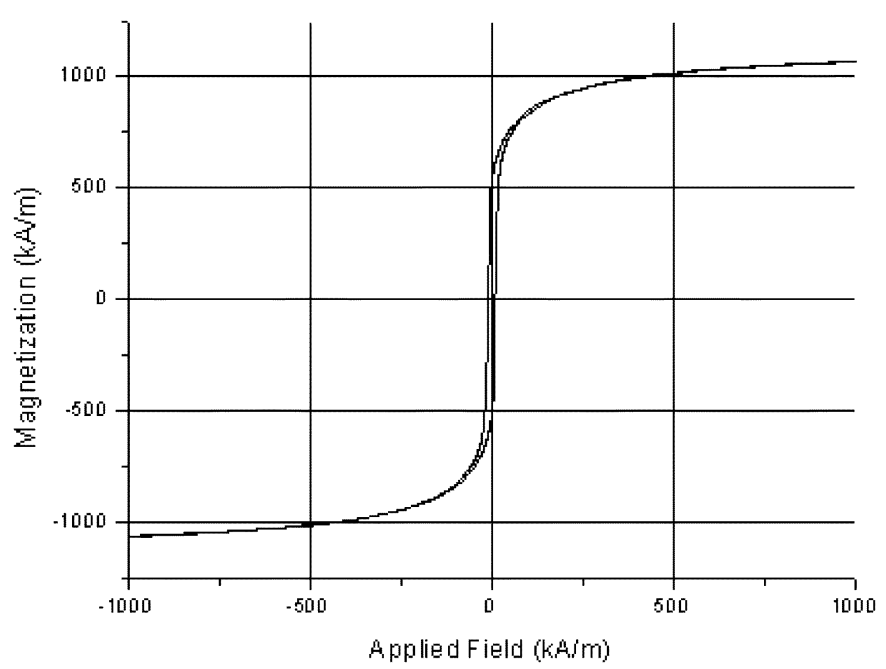

Fig. 4. Hysteresis curve $M$ versus $H$ for the electroplated cobalt layer measured with a Vibrating Sample Magnetometer. The cobalt layer has a coercive force of $9 \mathrm{kA} / \mathrm{m}$, a saturation magnetization of $1100 \mathrm{kA} / \mathrm{m}$, and a remanence of $525 \mathrm{kA} / \mathrm{m}$

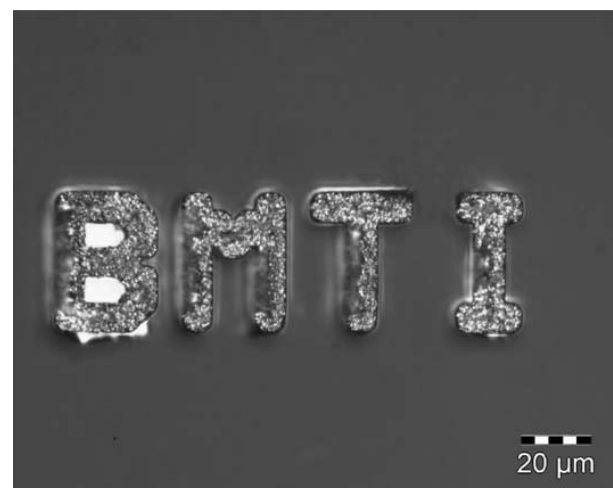

Fig. 5. Light microscopy image (in reflection), showing the versatility of the described delayed lift-off method. The electroplated cobalt structures shown are composed of lines of $11 \mu \mathrm{m}$ width, and have a thickness of $6 \mu \mathrm{m}$. The area surrounding the structures is bare glass. For very small enclosed structures, it can be difficult to remove the resist and seed layer. This can be seen in the letter " $\mathrm{B}$ " where the platinum seed layer is still present, which creates the strong reflection.

Fig. 4 shows typical $M$ versus $H$ hysteresis curve for the electroplated cobalt structures. The electroplated cobalt has a coercive force $H c$ of $9 \mathrm{kA} / \mathrm{m}$, a saturation magnetization of $1100 \mathrm{kA} / \mathrm{m}$, and a remanence of $525 \mathrm{kA} / \mathrm{m}$. The coercive force is very typical for these kinds of layers, while the remanence is quite high [13]. The saturation is at $75 \%$ of the maximum bulk cobalt saturation [11], which is good enough for our purposes, but can be further improved on.

Fig. 5 demonstrates the possibilities of the delayed lift-off method. The characters are produced with lines of $11 \mu \mathrm{m}$ width. The deposited cobalt has a thickness of $6 \mu \mathrm{m}$. Because of the method used, the area around the characters is just bare glass, devoid of any seed-layer, without any etching step. The achievable resolution of the structures produced is primarily determined by the resolution of the combination of photo resists and mask aligner that is used. For the smallest structures the strength of the connection to the glass is also important. The lift-off is a relative brute step that can pull these structures from the glass

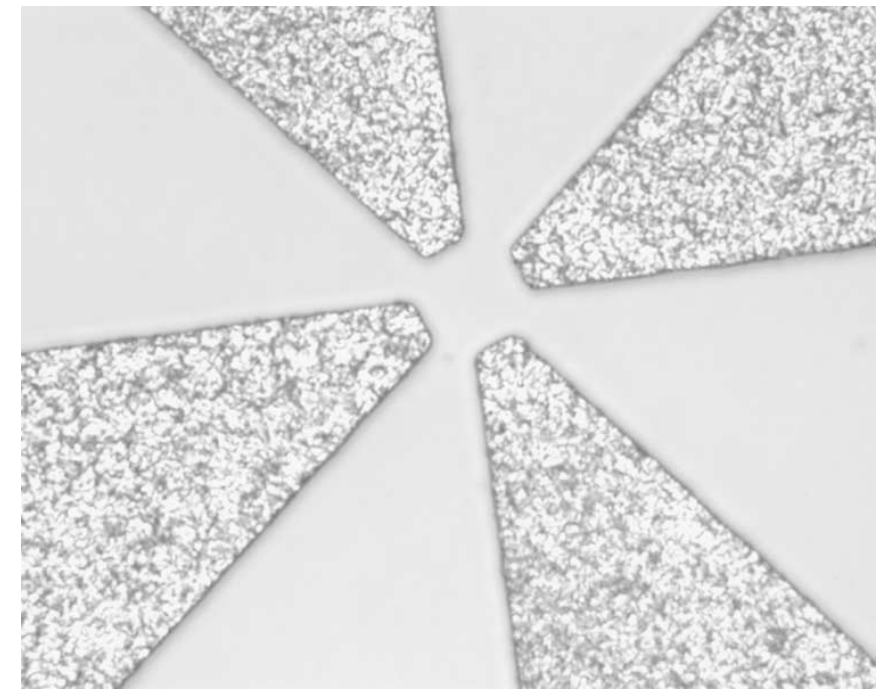

Fig. 6. The pole structures necessary for the magnetic tweezers. The pole tips are $4 \mu \mathrm{m}$ wide, and have a spacing of $20 \mu \mathrm{m}$, which allows the placement of a single cell in between them. The thickness of the poles is $6 \mu \mathrm{m}$, with a surface roughness of $0.5 \mu \mathrm{m}$.

when the connection to the surrounding seed-layer is stronger than the connection to the glass surface.

For biological application a magnetic structure with four poles is fabricated, as shown in Fig. 6. The pole tips are 4- $\mu \mathrm{m}$ wide, and have a spacing of $20 \mu \mathrm{m}$, which allows the placement of a single cell in between them. The thickness of the poles is $6 \mu \mathrm{m}$, with a surface roughness of $0.5 \mu \mathrm{m}$. These pole structures have been used to exert directional forces on magnetic beads.

\section{B. Force Measurements}

The magnetic force on a bead that was held at a fixed distance from the pole tips was measured as a function of the current through the coils. The results are shown in Fig. 7. The bead is held at a distance of $5 \mu \mathrm{m}$ from the attracting pole. The applied magnetic field is increased by increasing the current through the driving coils. The force on the bead shows a saturation behavior toward $3 \mathrm{nN}$ that can be ascribed to the saturation magnetization of the cobalt pole tips. The achieved forces are comparable to what can be achieved with single pole tip magnetic setups [1], and are an order of magnitude higher than setups that can apply directional forces [7].

\section{CONCLUSION}

A new method has been developed that allows patterned electro deposition on glass substrates. This "delayed lift-off" method allows for freestanding structures without a (residue) seed-layer on the glass surrounding them. The method uses standard clean room equipment, and is fast and easy to implement.

The method has been applied to create micrometer size magnetic structures that allow to exert directional magnetic forces on beads. Forces up to a few $\mathrm{nN}$ could be observed for $4.5 \mu \mathrm{m}$ Dynabeads. These results are promising for the development of new magnetic tweezers especially for biophysical research. 


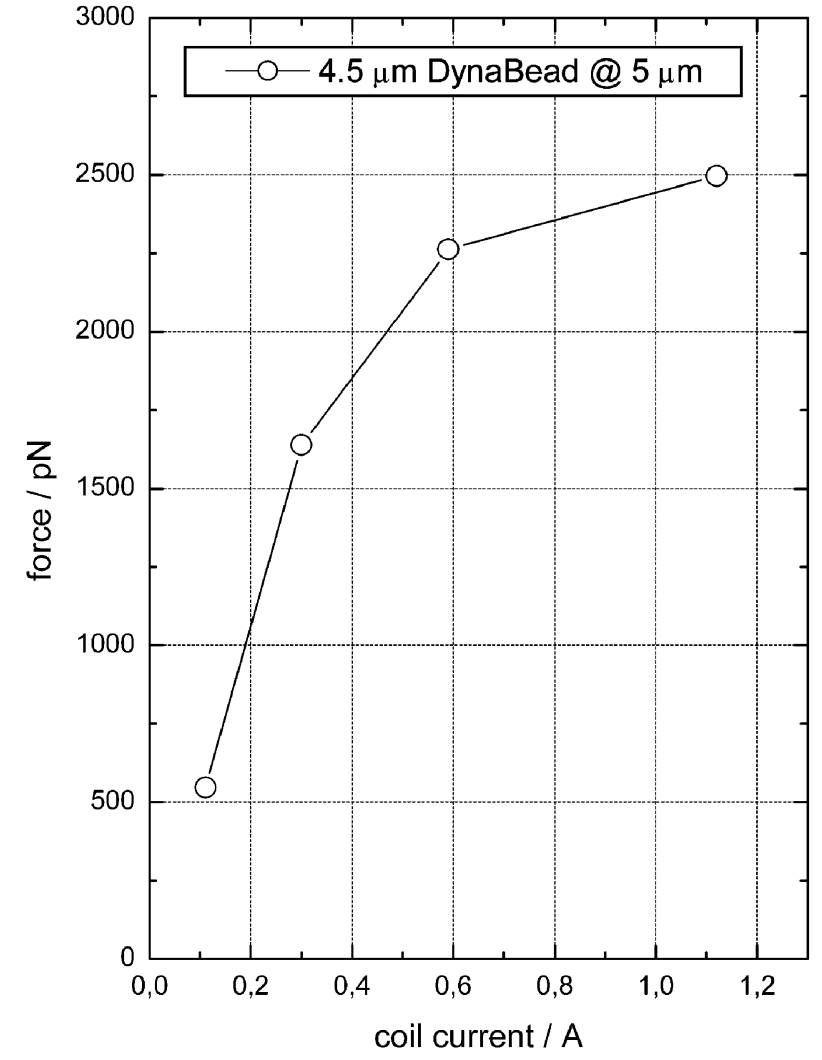

Fig. 7. Measurement of the magnetic force on a bead in the magnetic tweezers. An M-450 Dynabead is held at $5 \mu \mathrm{m}$ distance from the attracting pole and the current through the coils is gradually increased. At $2.5 \mathrm{nN}$, the force reaches its maximum because the pole tips become magnetically saturated.

This technology is currently used to fabricate more advanced magnetic structures that will allow 2-D manipulation of small beads inside living cells.

\section{REFERENCES}

[1] A. Bausch, F. Ziemann, A. A. Boulbitch, K. Jacobson, and E. Sackmann, "Local measurements of viscoelastic parameters of adherent cell surfaces by magnetic bead microrheometry," Biophys. J., vol. 75, pp. 2038-2049, 1998.

[2] D. Choquet, D. P. Felsenfeld, and M. P. Sheetz, "Extracellular matrix rigidity causes strengthening of integrin-cytoskeleton linkages," Cell, vol. 88, pp. 39-48, 1997.

[3] M. Radmacher, M. Fritz, C. M. Kacher, J. P. Cleveland, and P. K. Hansma, "Measuring the viscoelastic properties of human platelets with the atomic force microscope," Biophys. J., vol. 70, pp. 556-567, 1996.

[4] C. Pasternak, S. Wong, and E. L. Elson, "Mechanical function of dystrophin in muscle cells," J. Cell Biol., vol. 128, pp. 355-361, 1995.

[5] P. A. Valberg and H. A. Feldman, "Magnetic particle motions within living cells. Measurement of cytoplasmic viscosity and motile assay," Biophys. J., vol. 52, pp. 551-569, 1987.

[6] A. Bausch, W. Möller, and E. Sackmann, "Measurement of local viscoelasticity and forces in living cells by magnetic tweezers," Biophys. J., vol. 76, pp. 573-579, 1999.

[7] H. Huang, C. Y. Dong, H. Kwon, J. D. Sutin, R. D. Kamm, and P. T. C. So, "Three-dimensional cellular deformation analysis with a two-photonmagnetic manipulator workstation," Biophys. J., vol. 82, pp. 2211-2223, 2002.

[8] W. Ruythooren, K. Attenborough, S. Beerten, P. Merken, J. Fransaer E. Beyne, C. van Hoof, J. De Boeck, and J. P. Celis, "Electrodeposition for the synthesis of microsystems," J. Micromech. Eng., vol. 10, pp. 101-107, 2000.

[9] P. C. Andricacos, C. Uzoh, J. O. Dukovic, J. Horkans, and H. Deligianni, "Damascene copper electroplating for chip interconnections," IBM J. Res. Dev., vol. 42, pp. 567-74, 1998 .

[10] M. Datta and D. Landolt, "Fundamental aspects and applications of electrochemical microfabrication," Electrochimica Acta., vol. 45, pp. 2535-2558, 2000

[11] Cobalt, Metals Handbook, 9th ed., vol. 2, D. J. Maykuth, Ed., Metals and Ceramics Information Center, Battelle Memorial Institute, pp. 725-726.

[12] F. A. Lowenheim, Modern Electroplating, 3rd ed. New York: Wiley, 1974.

[13] S. Armyanov, "Crystallographic structure and magnetic properties of electrodeposited cobalt and cobalt alloys," Electrochimica Acta., vol. 45, pp. 3323-3335, 2000.

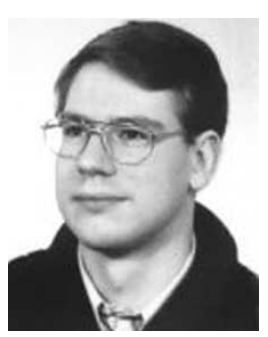

Anthony H. B. de Vries was born in 1972 . He received the applied phyiscs degree from the University of Twente, Enschede, The Netherlands. In 2000, he received the M.Sc. degree in applied physics on the subject of IR spectroscopy. He was offered a position as a Ph.D. student at the Biophysical Techniques Group at the University of Twente, to develop magnetic tweezers technology for nanomanipulation in living cells.

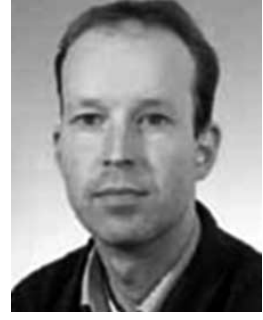

immuno sensors
Johannes S. Kanger received the M.Sc. degree in applied physics and the Ph.D. degree from the University of Twente, Enschede, The Netherlands, in 1992 and 1996, respectively.

From 1996 to 1998 , he worked as a Postdoctoral Fellow in the field of biomedical optics and noninvasive diagnostics. Since 1999, he has been working in the Biophysical Techniques Group at the University of Twente as a Senior Scientist in biophysics. His main research interests are single molecule detection and manipulation inside cells and integrated optical

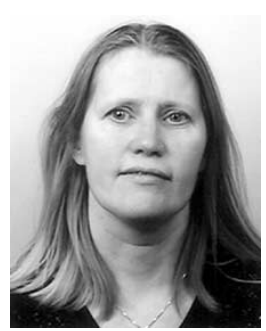

Bea E. Krenn was born in 1956. She studied chemistry at the Vrije Universiteit and specialized in biochemistry and microbiology. In 1985, she began pursuing the Ph.D. degree and performed research within the Department of Enzymology and Protein Structure at the University of Amsterdam. During this period she went to Japan for three months to study peroxidases from seaweeds at Kyoto University. In 1989, she defended her thesis: "Molecular aspects of bromoperoxidases from various species".

From 1989 to 1996, she performed biophysical and molecular biological research on membrane proteins, cyt bc complex and ATP synthase at the Vrije Universiteit. At the end of 2000, after giving birth to 2 children and 2 years as IT-specialist at IBM in The Netherlands, she started the biophysical work on the magneto-microscope described here.

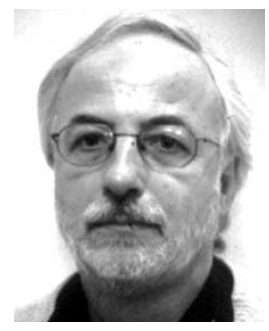

Roel van Driel was born in 1945 . He studied biochemistry at the University of Groningen in The Netherlands.

After receiving the Ph.D. degree at that university, he held a Postdoctoral position at the Biozentrum in Basel (Switzerland) and the Max-Planck Institute for Biochemistry in Martinsried, near Munich (Germany). After that, he moved to the University of Amsterdam and became full Professor at that university. His research group concentrates on the question how the eukaryotic genome is spatially organized inside the cell nucleus and how this affects and controls gene expression. Magnetic tweezers will be used to manipulate 3-D chromatin structure inside the nucleus and analyzing what the effect is on gene expression. 